innovare

Ciencia y Tecnología

\title{
ANÁLISIS DE LA SITUACIÓN ACTUAL DEL MODELO DE VINCULACIÓN DE UNITEC SAN PEDRO SULA ${ }^{1}$
}

\author{
José Isaac Domínguez Ortiz ${ }^{2}$ \\ Facultad de Ingeniería y Arquitectura, Universidad Tecnológica Centroamericana (UNITEC), \\ Campus de San Pedro Sula
}

(Recibido: Noviembre, 2017/ Aceptado: Enero, 2018)

\section{Resumen}

La extensión o vinculación universitaria se ha convertido en los últimos años, en una función sumamente preciada por cualquier universidad, principalmente porque funciona como ventaja competitiva para la misma, así como también permite establecer un vínculo con la sociedad y proyectar los conocimientos adquiridos en ella. El presente artículo busca analizar desde la perspectiva del proceso, cuál es la situación actual del modelo de vinculación de UNITEC, y ver cuáles son las limitantes que afronta el modelo actual, el nivel de calidad de los proyectos que se realizan y comparar el sistema con el de otras universidades de la región, con el fin de proponer mejoras que propicien, subsecuentemente, una mejoría en el sistema.

Palabras Claves: Extensión Universitaria, Diagrama de flujo, ONGs, Contrapartes, Vinculación, MiPymes.

\begin{abstract}
A community outreach program has become in recent years an asset for any university, mainly because it is understood as a competitive advantage. It fosters a different kind of link with society by allowing universities to project knowledge towards the community. This paper analyzes UNITEC's current community outreach model from the perspective of its process, in order to understand the constraints that it is actually facing as well as the quality of the projects that are actually carried out. This is done by comparing the in-house model with those used in other universities of the region, in order to propose improvements that could boost subsequent improvements.
\end{abstract}

Keywords: Community Outreach Program, Flowchart, NGOs, Counterparts, Micro, small and medium size businesses.

\footnotetext{
${ }^{1}$ El autor agradece a los Ingenieros Roberto Rodríguez (Coordinador de la Carrera de Ingeniería Industrial en el Campus de San Pedro Sula) y Andrea Orellana (Jefe de Vinculación en el Campus de San Pedro Sula), quienes le asesoraron y dieron importantes aportes en la metodología utilizada para desarrollar la presente investigación.

${ }^{2}$ Autor para correspondencia. Email: jisaac71196@unitec.edu
} 


\section{Introducción}

Desde hace mucho tiempo las funciones de las universidades han ido evolucionando constantemente. Lo que en un principio fue creado con el objetivo de formar personal calificado para el servicio de la iglesia, se fue transformando en grandes asociaciones en donde las personas transmitían conocimientos unos a otros. Las universidades se convirtieron en organizaciones para la enseñanza y el aprendizaje de una serie de disciplinas, las cuales poseían facultades como las artes, filosofía, derecho, medicina y teología. En ese entonces, solo las personas pertenecientes a altas clases sociales podían acceder a esa educación.

Según un artículo acerca de la evolución histórica de la universidad (Buchbinder, 2006), es a finales del siglo XVIII y principios del XIX en donde las universidades experimentaron nuevamente procesos de transformación. Fue a partir de esa época en donde las instituciones de educación superior comenzaron a desprenderse en forma definitiva de la influencia de la iglesia, se subordinaron a las autoridades civiles y se adecuaron a los cambios derivados del desarrollo de las ciencias y de las necesidades del Estado. A partir de ese hecho se empezó a exigir, de las casas de altos estudios, la generación de un conocimiento efectivamente útil para el desarrollo de la sociedad. Es ahí donde surge la génesis de la extensión universitaria, función que se fue esparciendo por todas las universidades de Europa.

Hoy en día las universidades no solamente tienen como función la transmisión de conocimientos, sino también la extensión de estos en la sociedad. La extensión universitaria, también llamada vinculación, es una actividad desarrollada en el entorno de la universidad, en la cual se enfatiza la relación de ésta con la comunidad en la que está inmersa. La extensión universitaria es una función sustantiva de toda universidad, y Universidad Tecnológica Centroamericana (UNITEC) no es la excepción, desde su fundación en el año 1986, UNITEC siempre ha estado firme en su compromiso de crear vínculos con la sociedad, beneficiando a pequeñas empresas, organización sin fines de lucro, etc.

En el presente documento se pretende hacer establecer la situación actual del modelo de vinculación de UNITEC. La investigación realizada para ese efecto es de carácter cualitativa, y en ella se describe el modelo de vinculación utilizado por UNITEC, para posteriormente realizar un análisis de su situación actual desde el punto de vista de la ingeniería industrial. Dicha investigación también ha englobado aspectos tales como la calidad de los proyectos realizados y las limitantes que afronta el modelo. Agregado a lo anterior, también implicó llevar a cabo un análisis comparativo entre las universidades de la región, con el fin de mostrar el posicionamiento del modelo de vinculación actual en contraste con los de otras instituciones de educación superior. 


\section{Problema de Investigación}

La vinculación es una función de mucha importancia en toda universidad ya que en ella se ve reflejado el compromiso que tiene ésta con la sociedad. También permite a los estudiantes tener un acercamiento con la realidad que vive su comunidad, así como poner en práctica los conocimientos que han adquirido en sus años de estudio. En la actualidad existen diferentes modelos utilizados por las universidades para llevar acabo la vinculación, saber cómo funcionan estos, los resultados e impactos que generan, permite a las universidades poder realizar mejoras a los mismos.

Por lo tanto, esta investigación va orientada a poder explorar cuál es la situación actual de este proceso en UNITEC, verificar si el modelo utilizado está funcionando o cumpliendo con los objetivos del mismo, analizar las diferentes funciones que este realiza, descubrir cuáles son las limitantes o dificultades que afronta actualmente, indagar acerca del nivel de calidad que posee, así como también realizar un comparativo del modelo utilizado por UNITEC en contraste con otras universidades del sector de San Pedro Sula.

\subsection{Objetivos de investigación}

Realizar un análisis acerca del estado actual del modelo de vinculación permitirá mejorar el sistema de vinculación de la universidad y con ello, poder proyectarse a un nivel superior. Asimismo, buscar la forma de realizar un mayor impacto a la sociedad, de una forma más eficiente y dinámica. Por lo tanto, los objetivos de esta investigación son los siguientes:

1. Indagar acerca de las limitantes que aquejan el actual sistema de vinculación universitaria de UNITEC.

2. Analizar el nivel de calidad de los proyectos de vinculación durante el periodo comprendido del 2013 al 2017.

3. Realizar un análisis comparativo del modelo de vinculación universitaria de UNITEC en contraste con los modelos de otras universidades del país.

\section{Marco de Referencia}

\subsection{Extensión o vinculación universitaria}

Extensión y vinculación universitaria son términos que en muchas ocasiones se usan como sinónimos. La extensión universitaria, según la Universidad Nacional de La Plata (UNPL), en un artículo publicado en Julio 2015, se define como "la presencia e interacción académica mediante la cual la Universidad aporta a la sociedad los resultados y logros de su investigación y docencia, y por medio de la cual, al conocer la realidad nacional, enriquece y redimensiona toda su actividad académica conjunta". 
Otra definición útil es la Bernheim (2003):

La extensión universitaria es la interacción entre la Universidad y los demás componentes del cuerpo social, a través de la cual esta asume y cumple su compromiso de participación en el proceso social de creación de la cultura y de liberación y transformación radical de la comunidad nacional (p.76).

Ahora bien, al referirse a la vinculación universitaria, García Matías, Morales Soto, \& Castellanos Suárez (2009) la definen señalando que:

Es una actividad que indica acción interactiva entre sujetos vivos (los objetos no pueden interactuar sin manipulación), por otra parte, este interactuar se presenta con ligas subjetivas no observables que pueden ser sentimientos, motivos, valores, intereses o correspondencias de persona a persona.

En lo que a este documento se refiere, también se utilizarán ambos términos como sinónimos, puesto que conllevan un funcionamiento en conjunto.

\subsection{Origen de la extensión universitaria}

En el siglo XIX es donde empieza a surgir lo que hoy se conoce como la tercera función sustantiva de la universidad: extensión o vinculación universitaria. Es así como aparecen los primeros intentos de acercar el conocimiento a la sociedad en general, sin excluir a las personas de bajo nivel económico, buscando con ello, solucionar los distintos problemas que aquejaban a la sociedad en aquel entonces.

Según D'Andrea et al. (2014), los primeros programas formativos para adultos se dieron en el año 1790, en Inglaterra, ante las necesidades planteadas por la Revolución Industrial. El primer colegio de clase obrera, orientado a satisfacer las necesidades educativas y de capacitación de la comunidad se funda en 1842 en Sheffield, Inglaterra, el cual tenía como propósito ofrecer educación a jóvenes de la clase trabajadora. Es así como las personas de bajos recursos empezaron a tener acceso a la educación universitaria.

Años después, en 1867 se estableció, en la Universidad de Cambridge, el primer programa de extensión universitaria. D’Andrea et al. (2014) describieron este programa como un sistema que reconocía la responsabilidad de las universidades hacia los sectores de bajos recursos, y que se extendió luego a otras universidades del país. El programa utilizaba centros en diversos pueblos como medio para la expansión del conocimiento. Cada pueblo solicitaba a Cambridge un curso específico de acuerdo con las necesidades latentes de ese pueblo y la universidad lo organizaba con la estructura de doce conferencias, estas eran dictadas por un maestro elegido por la institución, quien podría ser un docente o un recién egresado. El objetivo fundamental de estos programas de extensión era acercar el conocimiento al pueblo, abandonado el precepto que las universidades solo son para ricos.

Es a finales del siglo XIX que la extensión universitaria se fue extendiendo por toda Europa de forma constante, así como también da inicio en Estados Unidos. 


\subsection{La Extensión universitaria en Honduras}

Murillo (2017), realizó una investigación acerca de la educación superior en Honduras, en la cual menciona que el movimiento estudiantil cordobés de 1918, incidió en una nueva ideología de la universidad latinoamericana, así como también en la agenda política del movimiento estudiantil universitario hondureño, sobretodo en su búsqueda de una reforma universitaria para la Universidad de Honduras principalmente en la década de 1950.

Los postulados de Córdoba influyeron también en las proclamas y el accionar de las organizaciones estudiantiles de secundaria y de algunos partidos políticos. Un ejemplo de ellos es la FEUH (Federación de Estudiantes Universitarios de Honduras), fundada en el año 1925, la cual agrupó organizaciones políticas y asociaciones estudiantiles de las facultades de Derecho, Medicina e Ingeniería. Su vida activa como organización inició en 1929 con la elección de nuevas autoridades estudiantiles y con ellas la integración del Primer Comité Pro-Reforma Universitaria en 1931, en la cual se impulsó un proyecto de ley universitaria inspirado en los postulados de Córdoba.

Después de tantas luchas, huelgas y cambios políticos, finalmente se logró conseguir la autonomía universitaria el 15 de octubre de 1957, con la Junta Militar de Gobierno que integraban el coronel Héctor Carracioli y el mayor Roberto Gálvez Barnes. Mediante el Decreto número 170 se estableció Ley Orgánica de la Universidad Autónoma de Honduras, que estuvo vigente hasta el 11 de febrero de 2005. La Junta Militar de Gobierno reconoció la importancia de la autonomía para la Universidad de Honduras, y la consideró indispensable para el cumplimiento de objetivos en el desarrollo de la educación, la cultura y la ciencia.

Según Murillo (2017), entre los beneficios que se pueden denotar a partir de la autonomía universitaria están: poder elegir las propias autoridades académicas sin injerencia del exterior y la libre administración presupuestaria y académica sin intervención por parte del Estado.

Igualmente, la FEUH impulsó a que la Universidad Nacional Autónoma de Honduras administrara el espacio de la educación superior en Honduras. Además, estableció el cogobierno universitario, el cual tenía la función de organizar, dirigir y desarrollar con carácter exclusivo la educación superior y profesional de Honduras. Este órgano era conformado por estudiantes, docentes, autoridades, colegios profesionales y trabajadores donde los estudiantes tenían una representación del $50 \%$ en todos los órganos de gobierno de la universidad, disposición que estuvo vigente hasta el año 2005.

El movimiento estudiantil universitario influyó definitivamente en la construcción de la universidad hondureña para la década de los 60's y 70's, la cual fue establecida con un fuerte compromiso sociopolítico hacia la realidad nacional existente. 


\subsection{Desafíos de la extensión universitaria}

Desde hace un siglo, son muchos los autores que plantean los desafíos y retos con los cuales se ha enfrentado la extensión universitaria, los que en la actualidad aún siguen vigentes. Por lo tanto, para ir dándoles respuesta, se requiere una actualización en el proceso que permita redefinir el objetivo principal de la extensión universitaria. Algunos de estos autores identifican desafíos tales como la discriminación de clases sociales, la falta de enfoque de las universidades, la falta de comunicación entre las contrapartes, la falta de inclusión en la estructura de las instituciones educativas, entre otros.

En el artículo “Misión Social y Modelos de Extensión” (Alcantara, 2007), el autor propone modelos que permiten el favorecimiento de las poblaciones marginadas y en los cuales se establecen procesos personales, grupales y comunitarios en la solución de problemas concretos, evitando el asistencialismo. Estos modelos deberán ser asumidos por la comunidad universitaria y deberán ser fundamentales en la formación de los estudiantes.

El Doctor Luis Llorens Baez (1992), sostiene la necesidad de planificar la extensión partiendo del reconocimiento de las condiciones concretas que determinan su ubicación real en la estructura y en el funcionamiento orgánico de la institución. Estableciendo su función en la estructura de la universidad y dándole la responsabilidad de consolidar a la misma de ser la casa de cultura.

\subsection{Modelos de extensión universitaria}

La extensión universitaria también se fue expandiendo en América Latina. Las universidades fueron adquiriendo diferentes modelos con diferentes enfoques en la sociedad, compartiendo siempre el mismo objetivo principal que es el de transferir los conocimientos a la sociedad.

Existen actualmente una variedad de modelos que utilizan las distintas universidades del mundo. En el artículo escrito por Ortiz-Riaga \& Morales-Rubiano (2011), los autores describen cuatro modelos que se cree iniciaron en el siglo XX:

a) Altruista: este modelo prevaleció en las primeras décadas del siglo y concebía la extensión como las acciones desinteresadas de los universitarios en favor de las poblaciones marginadas o sectores de bajo nivel económico.

b) El divulgativo: el cual consistía en acercar a la población los adelantos científicos y técnicos, así como también a las expresiones culturales producidas por la universidad.

c) El concientizado: consistía en una visión que persigue la creación de conciencia, el despertar de la capacidad de análisis críticos y la acción eficaz y transformadora, lo que desemboca en una participación política activa y en la constitución de grupos de interés y de presión que van en contravía de la burocracia institucional.

d) El vinculatorio empresarial: un modelo el cual iba enfocada a satisfacer las necesidades de las empresas. Este modelo toma fuerza a mitad de la década de los 
ochenta, y tuvo como consecuencia que la universidad comenzó a percibir beneficios económicos por sus servicios.

\subsection{Objetivos de la extensión universitaria}

Según Tünnermann Bernheim (2003, pp.76-77) la extensión universitaria en términos generales tiene como objetivos fundamentales:

1. Contribuir a la creación de una conciencia crítica en todos los sectores sociales del país o sector involucrado, esto para favorecer un verdadero cambio liberador en la sociedad.

2. Contribuir a que todos los sectores alcancen una visión integral y dinámica del hombre y el mundo, en el cuadro de la realidad histórico-cultural y del proceso social de emancipación de la América Latina.

3. Promover como integradora de la docencia y la investigación la revista crítica de los fundamentos de la universidad y la concientización de todos sus estamentos, para llevar adelante un proceso único de creación cultural y trasformación social.

4. Contribuir a la difusión y creación de los modernos conceptos científicos y técnicos que son imprescindibles para lograr una efectiva transformación social.

Estos objetivos se basan principalmente, y de forma general, en la definición de extensión universitaria. No son objetivos representativos de todos los modelos existentes utilizados por las universidades, pero son objetivos que las universidades deben tomar en cuenta o basarse en ellos para la creación de los propios dependiendo del estado social de cada país.

\section{Metodología}

\subsection{Enfoque y métodos}

El presente trabajo es una investigación descriptiva-cualitativa, Su método es de carácter exploratorio. Busca examinar la situación actual de la vinculación de UNITEC, y la realización de un análisis acerca de sus dificultades, calidad e impacto que posee el modelo actualmente, con el fin de proponer mejoras para el mismo.

Para la elaboración de la investigación se utilizaron diversas herramientas para recopilar información, pero la "entrevista a expertos" fue la técnica principalmente escogida para la recolección de estos datos.

\subsection{Técnica aplicada}

La primera herramienta de recolección de datos es la entrevista, el tipo de entrevista que se realizará es semiestructurada, lo cual significa que se llevará a cabo siguiendo una guía de preguntas, las cuales están sujetas a cambios o adiciones, dependiendo de cómo se lleve a cabo la entrevista, con el fin de obtener más información sobre el tema abordado. Se escogió la entrevista como herramienta de recolección de datos debido a que permite 
establecer un flujo de información directo, recibiendo los datos de forma precisa y detallada.

Se realizaron dos tipos de entrevistas. La primera fue hecha tanto a expertos, como a estudiantes de UNITEC. Los expertos entrevistados fueron las personas encargadas del departamento de Vinculación y Emprendimiento de UNITEC. El propósito de dirigir a ellos esta entrevista fue la de obtener información precisa, extremo que se previó se lograría gracias a los años de experiencia de los entrevistados en el tema estudiado. Agregados También se realizaron entrevistas a 20 estudiantes de UNITEC, pertenecientes a diferentes carreras, con el objetivo de conocer su opinión acerca del proceso analizado, así como para conocer las limitantes que han experimentado en el desarrollo de sus proyectos.

El segundo tipo de entrevista que se utilizó fue la entrevista telefónica, dirigida a representantes de algunas de las contrapartes de UNITEC que se han visto beneficiadas por el servicio de vinculación, con el fin de conocer el nivel de calidad de los proyectos que se han realizado y el grado de aplicabilidad de los mismos.

\subsection{Fuentes de información}

Para desarrollar esta investigación se utilizaron diversas fuentes de información. Las fuentes primarias que se utilizaron fueron la del personal del departamento de Vinculación e Emprendimiento de UNITEC. Como fuentes secundarias de información, se utilizaron artículos educativos, libros, revistas científicas, información directa de las páginas de universidades que realizan vinculación en Latinoamérica, así como también casos de éxito.

\section{Resultados y Análisis}

Para comenzar con el análisis de la situación actual del modelo, es preciso describir el funcionamiento actual del mismo, características, tipos de programas, organizaciones con las que laboran, entre otros aspectos.

La Universidad Tecnológica Centroamérica (UNITEC), es una institución privada de educación superior, que fue creada el 17 de diciembre de 1986, con el propósito de convertirse en una alternativa para la formación universitaria, tanto por su innovadora oferta académica, como por su propuesta y modelo educativo. UNITEC cuenta con varios departamentos que cumplen diferentes funciones, entre las que se encuentra "Vinculación" el área encargada de involucrar actividades orientadas a beneficiar a poblaciones a través de programas, investigaciones, etc.

El modelo básico de vinculación de UNITEC abarca diferentes tipos de proyectos que pueden ser desarrollados ya sea como práctica profesional, voluntariado, clases, proyectos especiales, siempre que la universidad considere que estos permitan el desarrollo humano y profesional de los estudiantes. El modelo define los beneficios que obtienen los estudiantes al realizar estos proyectos, los cuales se resumen en "aprender", "aplicar sus 
conocimientos" y "ayudar a otros". También establece cuáles son las organizaciones que benefician de ellos, pudiendo estas ser micro y pequeñas empresas, los gobiernos centrales, gobiernos locales, organizaciones de desarrollo, etc. Más adelante se muestra el tipo de clasificación que utiliza la universidad para poder agrupar las organizaciones con las que trabaja.

El principal programa o sistema por medo del cual se llevan a cabo los proyectos de vinculación de UNITEC se denomina ProSeSo, Programa de Servicio Social. En éste, cada alumno de UNITEC ha desarrollado 100 horas de servicio comunitario, participando en la ejecución de proyectos encaminados a resolver problemas que obstaculizan el desarrollo de las comunidades de Honduras. Actualmente el número de horas fue incrementad de 100 a 140 horas de vinculación para los estudiantes que se matricularon en el año 2017. Su cumplimiento es de carácter obligatorio, por lo tanto, el estudiante debe participar activamente en proyectos de vinculación, para ir acumulando horas, y alcanzar la cantidad mínima establecida.

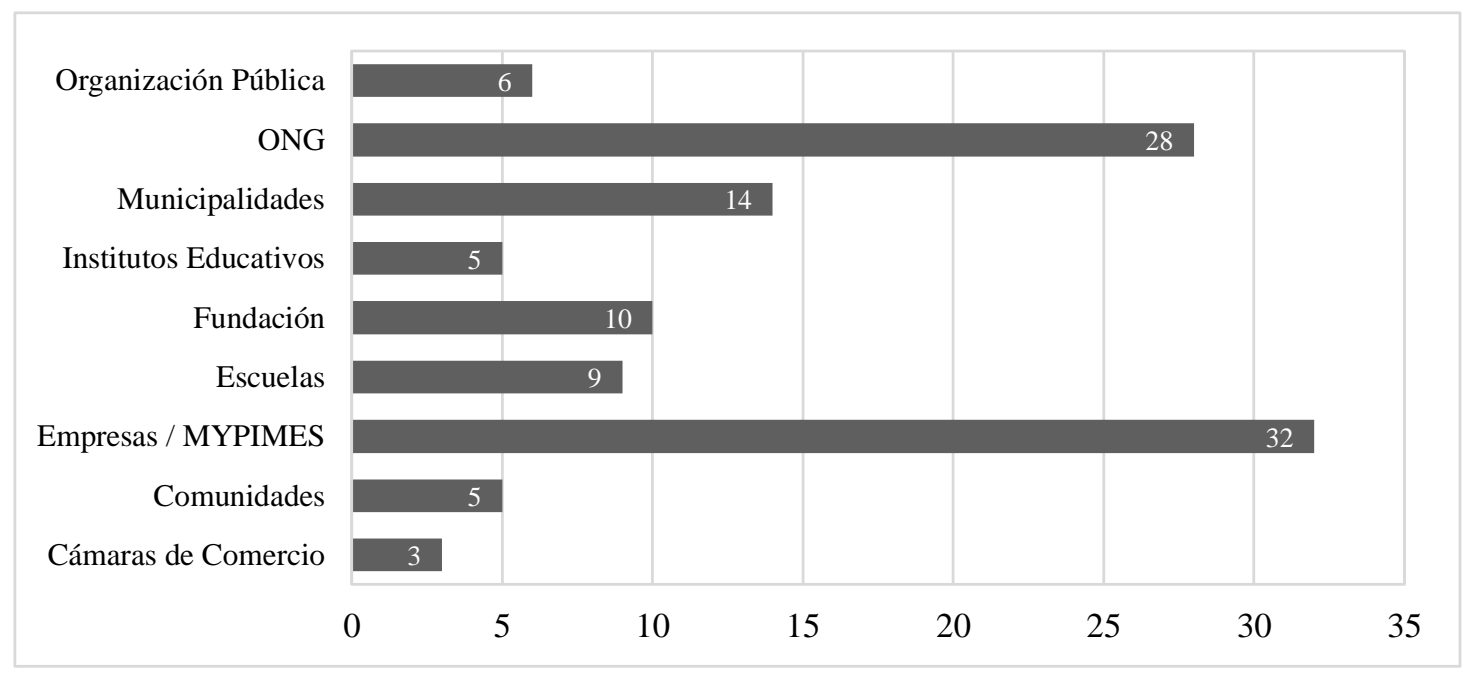

Figura 1. Tipos de Organizaciones Apoyadas

Fuente: Elaboración Propia con datos provistos por el Departamento de Vinculación de UNITEC San Pedro Sula

En la Figura 1, se muestran todos los tipos de organizaciones con las que trabaja UNITEC y la cantidad de organizaciones que fueron apoyadas durante el año 2016. En el gráfico es fácilmente apreciable, como las empresas/MiPymes y las ONG'S abarcan cerca del 53.57\% del total de organizaciones con las que se trabaja. También se benefician significativamente las municipalidades. en el año 2016 fueron beneficiadas 14, según la Figura 1.

De acuerdo a la información recopilada a través de las entrevistas a expertos, cada carrera de la universidad tiene establecida una serie de clases para las que la vinculación es obligatoria, y es deber del docente coordinar las actividades de vinculación con cada grupo matriculado en ellas. 


\subsection{Situación actual del proceso de vinculación de UNITEC}

En la actualidad no hay un proceso de vinculación claramente establecido en UNITEC. No obstante, tomando en cuenta la información recopilada en las entrevistas a expertos se realizó un diagrama de flujo con el fin de visualizar dicho proceso de forma más detallada, y poder posteriormente proponer mejoras al mismo. Para el diagrama de flujo se utilizó la simbología de las Normas ANSI, y se estableció un número correlativo en cada paso, con el propósito de facilitar su posterior análisis y explicación.

El proceso se subdivide en dos fases, la primera abarca la parte administrativa del proceso, iniciando con el contacto con la organización a beneficiar, hasta la transferencia del proyecto al docente. A partir de ahí, comienza la segunda fase que implica el desarrollo del proyecto.

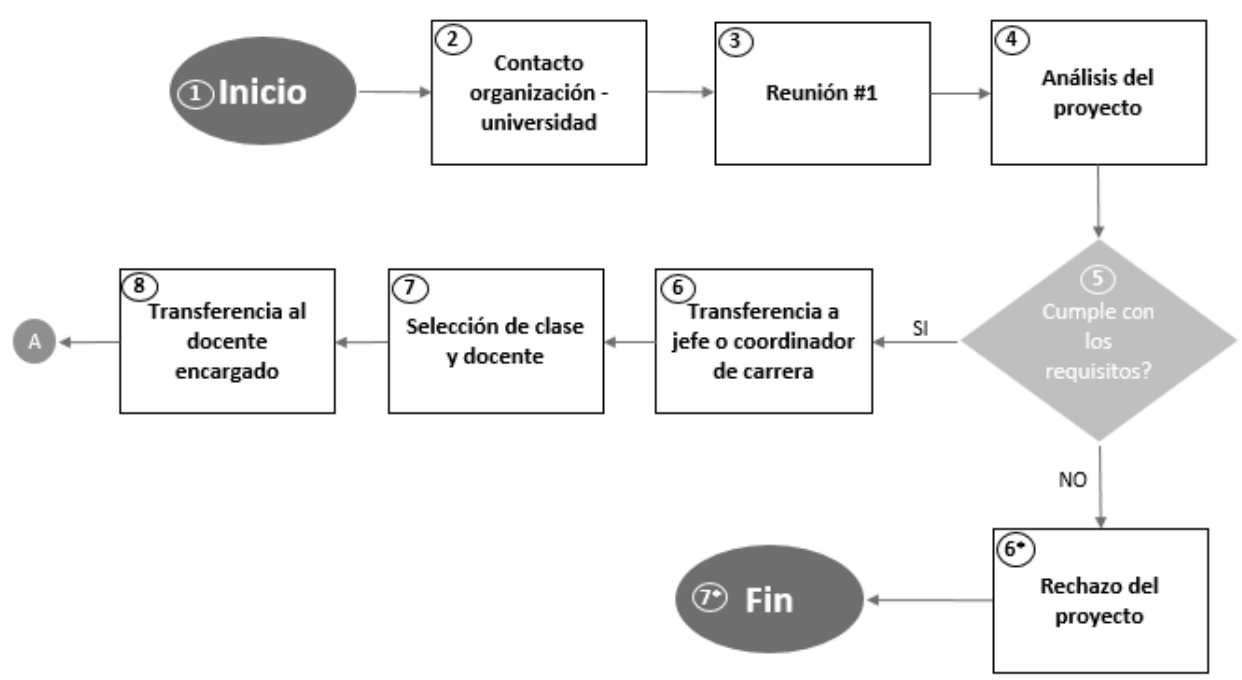

Figura 2. Diagrama de Flujo de la Primera Fase

Fuente: Elaboración Propia

El proceso se desarrolla de la siguiente forma:

- Paso \#2, que consiste en el primer acercamiento entre la organización que requiere el servicio de vinculación y la universidad.

- Paso \#3, donde se agenda una cita para realizar la primera reunión con el Jefe de Vinculación y Emprendimiento.

- Paso \#4, en el que el Departamento de Vinculación y Emprendimiento analiza el proyecto propuesto, y dependiendo de su tamaño y alcance, lo remite al Comité de Investigación, Vinculación y Emprendimiento (CIVE). Este comité evalúa el proyecto, para determinar si su ejecución es económicamente viable, si la organización es una micro, pequeña o mediana empresa o si es una organización sin fines de lucro. 
También se analiza la ubicación de la organización en cuestión de seguridad, entre otros aspectos.

- Paso \#5, el comité decide si el proyecto cumple o no con los requisitos. Si el proyecto es aprobado, continúa con el paso \#6 y si no, continúa con el paso \#6*.

- Paso \#6, el comité lo transfiere a el coordinador o jefe de carrera para que analice el proyecto

- Paso \#7, el coordinador asigna una clase en específico para realizar el proyecto.

- Paso \#8, el coordinador transfiere el proyecto al docente de la clase, quien se encargará de desarrollarlo en conjunto con los alumnos.

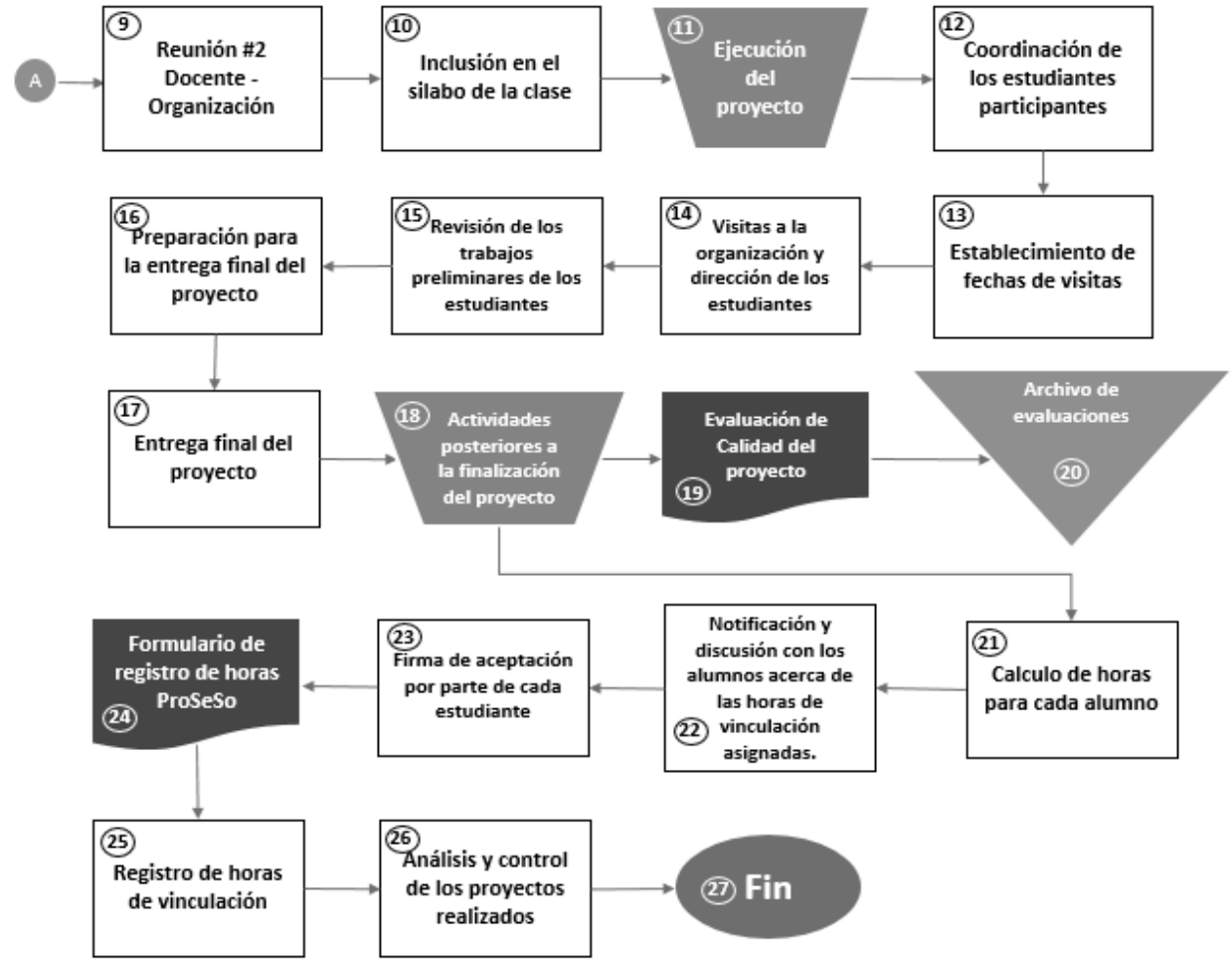

Figura 3. Diagrama de Flujo de la Segunda Fase.

Fuente: Elaboración Propia

La segunda fase del proceso se desarrolla de la siguiente manera:

- Paso \#9, una vez transferido el proyecto al docente, se establece una segunda reunión con la contraparte, con el objetivo de esclarecer dudas.

- Paso \#10, se incluye el proyecto en el sílabo de la clase.

- Paso \#11, comienza la etapa de ejecución del proyecto

- Paso \#12, el docente comienza con la coordinación de los estudiantes participantes.

- Paso \#13, de ser necesario, se establecen las fechas de las visitas de los proyectos. 
- Paso \#14, se continúa con el desarrollo del proyecto (visitas, investigaciones, etc).

- Paso \#15, consiste en la revisión de los trabajos preliminares de los estudiantes.

- Paso \#16, se establece lineamientos finales para la entrega del proyecto.

- Paso \#17, se realiza la entrega final del proyecto, en donde el docente y/o estudiantes muestran los resultados del mismo a la contraparte.

- Paso \#18, comienza la etapa posterior a la finalización del proyecto.

- Paso \#19, se evalúa la calidad general del proyecto y sus resultados.

- Paso \#20, el docente entrega las evaluaciones finales al Departamento de Vinculación y Emprendimiento, el que procederá a archivarlas.

- Paso \#21, el docente calcula las horas de vinculación que asignará a cada alumno.

- Paso \#22, el docente notifica a los estudiantes acerca de las horas que les han sido asignadas.

- Paso \#23, los estudiantes firman aceptando la cantidad de horas de vinculación asignadas.

- Paso \#24, se llena un formulario de registro de las horas de vinculación por parte del docente. En este formato se ingresa información relacionada con la contraparte, al nivel de calidad del proyecto desde el punto de vista del docente, etc.

- Paso \#25, el docente entrega los documentos al Departamento de Vinculación y Emprendimiento, el cual procede a registrar las horas de los alumnos participantes.

- Paso \#26, este departamento realiza diversos análisis y controles estadísticos de las horas de vinculación realizadas, los proyectos ejecutados, etc. Una vez realizado este paso, el proceso llega a su fin.

El proceso antes descrito es el que se lleva acabo con organización que entablan relaciones por primera vez con la universidad. No obstante, el proceso se puede llegar a tener variaciones, como, por ejemplo, en cuanto a la cantidad de reuniones realizadas para la definición del proyecto. Ha existido casos en los que se necesita realizar más de una reunión para definir el proyecto y establecer condiciones, etc. También puede haber variaciones en el proceso, dependiendo del tipo de proyecto a realizar, sobre todo si son proyectos como donaciones, celebraciones, etc. Para estos, se lleva a cabo un proceso más corto, debido a su naturaleza y simplicidad.

\subsection{Limitantes encontradas en el proceso}

Uno de los problemas con el proceso antes descrito se identificó en el Paso \#4, de donde se desarrolla la Reunión \#1, es que el análisis de los requisitos demuestra que ésta no se realiza de forma rigurosa, debido a que no existen requisitos establecidos para las organizaciones que soliciten el beneficio de vinculación. Se llegó a esta conclusión a partir de las entrevistas que se realizaron a los expertos y después de analizar el reglamento de vinculación y servicio social de la universidad. Los únicos requisitos que se les pide a las contrapartes es el apoyo en cuanto al transporte de los alumnos a las comunidades si fuera necesario, que la organización o comunidades beneficiarias con el servicio social realizado por los alumnos esté localizada a una distancia no mayor de $100 \mathrm{~km}$ del campus, y que 
llegar hasta ahí no implique un tiempo de viaje superior a 2 horas de ida y 2 horas de regreso. Un requisito adicional es que las contrapartes deberán ser entes que no posean la capacidad económico-intelectual para poder realizar el proyecto que solicitan. Sin embargo, este requisito no siempre es respetado, y de igual forma se permite se prosiga con la ejecución de los proyectos. Por lo tanto, a partir de este análisis se propone establecer algunos lineamientos que, si bien no han de ser excesivamente rigurosos, deberán permitan a la universidad enfocar su servicio a organizaciones que de verdad lo necesitan.

Otro problema identificado se da en el Paso \#5 "Análisis del proyecto". Esta parte del proceso, se supone va orientada a poder caracterizar adecuadamente el proyecto que se pretende desarrollar, así como las implicaciones que pueda conllevar, entre otros aspectos. Como se explicó anteriormente, dependiendo de la magnitud del proyecto, el Departamento de Vinculación y Emprendimiento lo remite al CIVE, el cual se encarga de analizar otros aspectos del mismo.

No obstante, lamentablemente, cuestiones como la seguridad en las visitas son aspectos que no siempre se analizan con rigurosidad a pesar de tener estos dos filtros, por lo tanto, el alumno muchas veces queda expuesto a los peligros de las zonas que visita. Según las entrevistas a expertos realizadas en el año 2016, se dio un asalto a un estudiante en el lugar donde se llevó acabo la vinculación. También informaron los expertos que en lo que va del año 2017, han ocurrido dos asaltos en las zonas donde se realizan las visitas. Esto lleva a la conclusión que es imperativo establecer lineamientos para el análisis de los proyectos de vinculación, en donde se establezcan los aspectos que obligatoriamente deben ser evaluados.

También se identificó un problema en el Paso \#10, de "Inclusión en el sílabo de la clase". Según las entrevistas a expertos, el objetivo de este paso es establecer aquellas clases en las que deberán realizarse actividades de vinculación, de esa forma los estudiantes que se matriculen, tendrán la oportunidad de participar en proyectos y acumular las horas de vinculación obligatorias. No obstante, esto no es garantía de que en dichas clases se lleven a cabo realmente actividades de vinculación, incidiendo en que los estudiantes no acumulen suficientes horas.

En este caso, la solución va orientada a que sean los docentes los que establezcan el contacto con las organizaciones con las cuales podrían desarrollar estos proyectos, procurando que estos puedan desarrollarse de manera continua. También se considera pertinente que se incentive a los alumnos a proponer ellos mismos posibles proyectos de vinculación. La definición de estos proyectos debe ocurrir antes del inicio del trimestre para contar con más tiempo para su ejecución.

Además de los problemas observados, es necesario señalar que algunas de las etapas de este proceso se pueden considerar superfluas, debido a que se realizan sin un propósito determinado y no impactan significativamente los objetivos del Departamento de Vinculación y Emprendimiento. Un ejemplo de lo anterior es el Paso \#19, de "evaluación de calidad del proyecto". Con este paso se pretende medir el nivel de calidad del proyecto 
recibido por las contrapartes, una vez que ha sido ejecutado. Esta actividad se lleva a cabo por el docente, sin embargo, la universidad no analiza esta información posteriormente. Si se analizaran las evaluaciones que se han llevado a cabo, la información resultante podría servir para realizar mejoras al proceso y mejorar al mismo Departamento de Vinculación y Emprendimiento.

También se identificó que hay problemas en el registro de las horas, pues según las entrevistas a los estudiantes, existen casos en los que no se registraron las horas que correspondían a los estudiantes de manera adecuada. Por lo tanto, se propone establecer herramientas como Excel de forma obligatoria para la recopilación de los datos de los estudiantes.

En resumen, se puede decir que la mayoría de los problemas del proceso analizado se deben más a una falta de compromiso en la ejecución del mismos. Por lo tanto, se propone establecer lineamientos obligatorios para las etapas de "Análisis de proyecto", "Inclusión en el sílabo de la clase", "Acompañamiento y dirección de los estudiantes" y "Firma de aceptación por parte de cada estudiante".

\subsubsection{Diagrama de flujo modificado.}

El siguiente diagrama muestra algunas modificaciones realizadas al proceso, como una propuesta de mejora, orientada principalmente a tener un enfoque hacia la calidad de los proyectos realizados, dando mucho más énfasis al análisis de las evaluaciones hechas por los participantes.

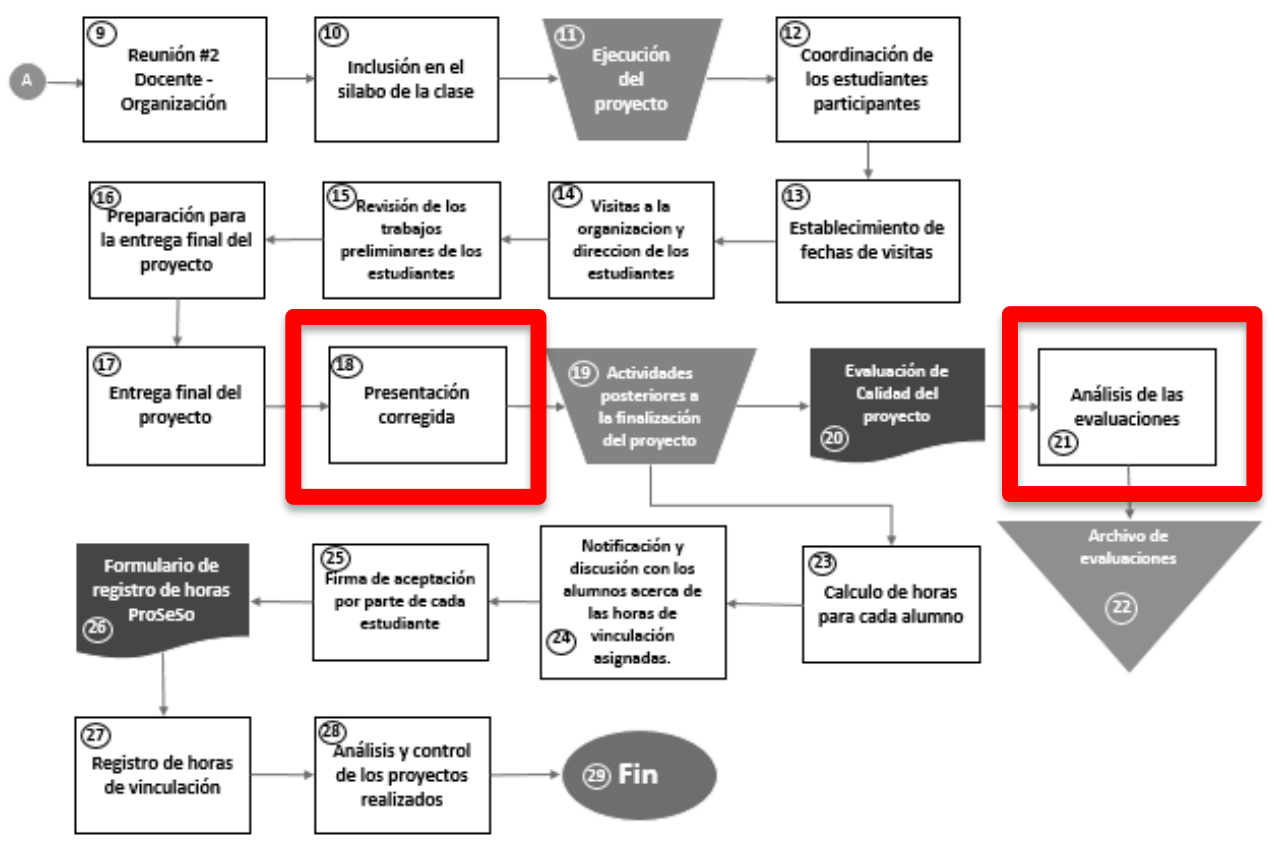

Figura 4. Diagrama de Flujo Propuesto

Fuente: Elaboración Propia 
En el diagrama se agregan dos pasos más que son los siguientes:

- Paso \#14, de "Presentación corregida", este va destinado a corregir cualquier error o problema que haya sido visto por el beneficiario del proyecto en la presentación final del mismo.

- Paso \#21, de “Análisis de las evaluaciones", el cual va destinado a analizar la información recopilada por las evaluaciones de calidad aplicada a los beneficiarios, para poder darles una voz a estos, así como también permitirles aportar sugerencias para el mejoramiento del proceso en la universidad.

\subsection{Limitantes encontradas en la etapa de desarrollo de los proyectos de vinculación.}

Para obtener un análisis más profundo y generalizado acerca de las limitantes que existen en el proceso de vinculación, se entrevistó a 20 estudiantes pertenecientes a diversas carreras de la universidad, con el fin de conocer su opinión acerca del proceso actual del modelo de vinculación, los beneficios que perciben de ella, limitaciones que han encontrado a lo largo de su vida universitaria, entre otros aspectos. En la siguiente gráfica se puede ver las limitantes mencionadas por los estudiantes. Cabe destacar que la selección de los participantes en la entrevista fue hecha de forma aleatoria, para obtener información real de estos aspectos.

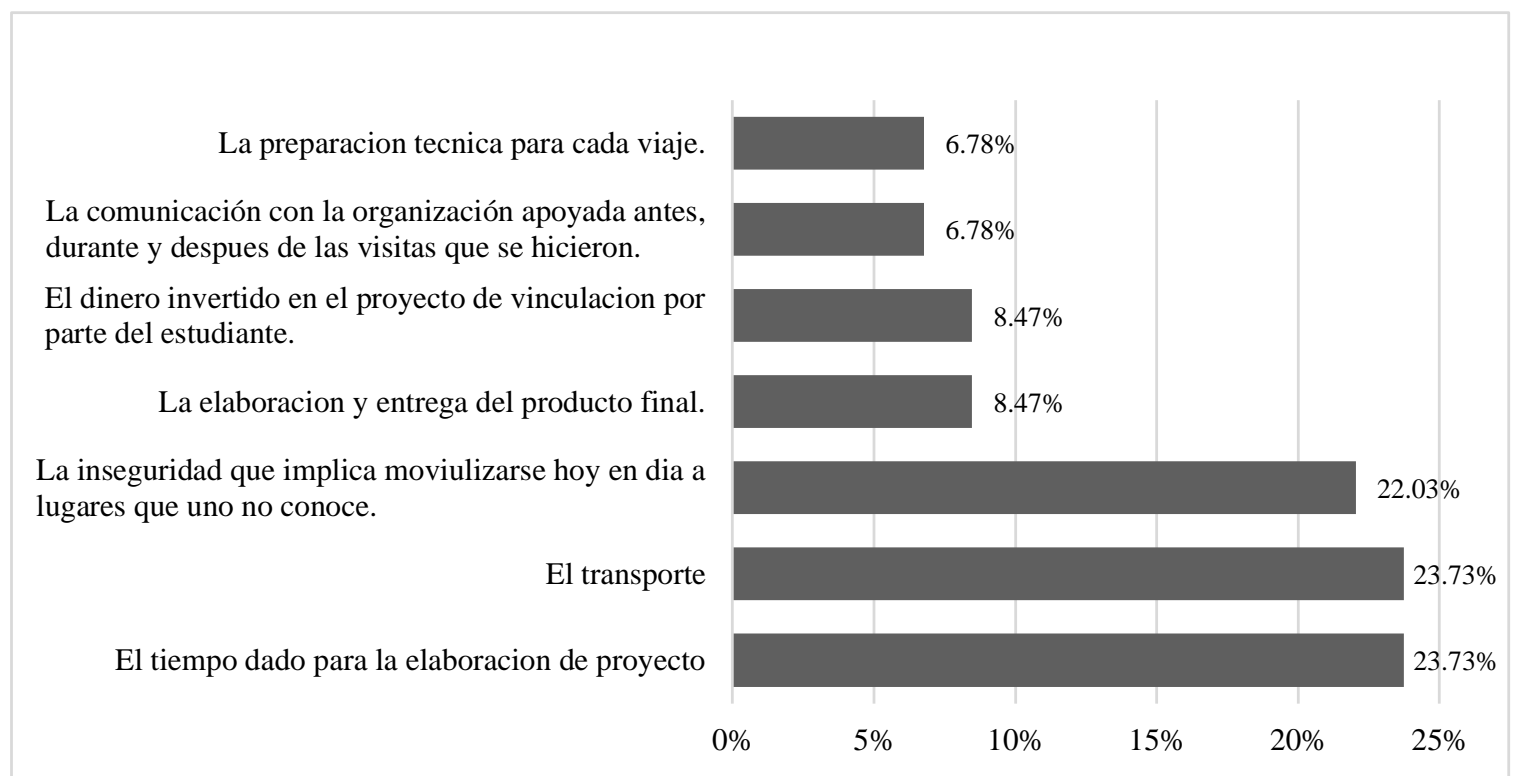

Figura 5. Limitantes encontradas en la etapa de desarrollo de los proyectos

Fuente: Elaboración Propia

Según los datos recopilados de las entrevistas a los estudiantes, aspectos tales como el tiempo, el transporte y la inseguridad son las mayores limitantes a la hora de realizar el proceso de vinculación (Figura 5). Juntas, estas variables equivalen al 69.49\% del total de las limitantes identificadas por los estudiantes. En un orden descendente, los resultados para cada una fueron: el $23,73 \%$ de los entrevistados mencionó al tiempo como una 
limitante encontrada en el proceso; el 23,73\% de los encuestados seleccionó el transporte como una limitante encontrada; y, el 22,03\% de los entrevistados mencionó la inseguridad de las zonas en donde se realizan los proyectos de vinculación.

\subsubsection{Relación Vinculación- Carrera.}

Para continuar con el análisis, se realizó una relación entre la carrera a la cual pertenecen los estudiantes entrevistados y su opinión respecto a si los proyectos de vinculación, tratando de entender si los proyectos que han realizado han estado orientados a su carrera de estudio. El propósito de lo anterior es entender la perspectiva del estudiante en cuanto al enfoque de estos proyectos y así, establecer si este aspecto puede llegar a convertirse en una limitante, pues al no estar orientada esta actividad al desarrollo de sus futuras habilidades profesionales, no estaría cumpliendo a cabalidad con los aspectos de generación de competencias en los estudiantes.

Al preguntarles sobre la relación vinculación-carrera, muchos comentaron que las actividades que han desarrollado no tiene relación en absoluto con su carrera, otros mencionaron que sí la tenía, y otros revelaron que la mayoría de sus proyectos si han tenido relación con su carrera, mientras que hubo quienes mencionaron que la cantidad de proyectos que han realizado con relación a su carrera es muy poca. Las respuestas de los estudiantes fueron agrupadas de la siguiente manera: "No", "Si", "Muy Poca", "La mayoría".

Los porcentajes de respuestas obtenidas de los estudiantes pertenecientes a la Facultad de Ingeniería y Arquitectura son los siguientes:

Tabla 1. Resultados de la Encuesta a Estudiantes sobre la relación que hay entre las actividades de vinculación y su carrera.

\begin{tabular}{|l|c|c|}
\hline \multicolumn{1}{|c|}{ Relación } & $\begin{array}{c}\text { Facultad de Ingeniería y } \\
\text { Arquitectura }\end{array}$ & $\begin{array}{c}\text { Facultad de ciencias } \\
\text { administrativas y sociales }\end{array}$ \\
\hline ALTA & $47 \%$ & $18 \%$ \\
\hline MEDIA & $0 \%$ & $27 \%$ \\
\hline BAJA & $21 \%$ & $9 \%$ \\
\hline SIN RELACION ALGUNA & $32 \%$ & $46 \%$ \\
\hline
\end{tabular}

Fuente: Elaboración Propia

En este caso el $55 \%$ de los encuestados mostraron una respuesta negativa acerca del tema abordado. Nuevamente se puede ver que en la Facultad de Ciencias Administrativas y Sociales hay un porcentaje mayor de personas que dijeron "No" en comparación con la Facultad de Ingeniería y Arquitectura, por lo que podemos concluir con que el problema podría tener una mayor incidencia en la Facultad de Ciencias Administrativas.

Del total de alumnos entrevistados, se agruparon las respuestas por carrera. Pudimos denotar varias carreras en donde había más incidencias de respuesta "NO" y "Muy pocos". Se indagó acerca de las posibles causas de estas respuestas. 
La primera carrera fue "Administración Industrial y de Negocios", en donde el $60 \%$ de los entrevistados mencionó que del total de proyectos que han realizado hasta el momento, muy pocos han tenido relación con su carrera universitaria. Entre las causas que se pueden atribuir a este resultado es la dificultad de encontrar proyectos que vayan acorde con dicha carrera. La mayoría de los entrevistados mencionó que los proyectos que mayormente se ejecutan son donaciones, celebraciones, etc., con organizaciones caritativas, así como eventos especiales de la universidad.

Otra de las carreras analizadas es la de "Marketing y Negocios Internacionales", en donde la mayoría de las respuestas se repartieron entre SI y NO. El 67\% de los alumnos mencionó que no hay relación entre los proyectos que han realizado, y el resto mencionó que si hay relación entre los dos objetos de estudio. En este caso, los estudiantes mencionaron causas similares a las comentadas para la carrera antes comentada, así como el enfoque que se da a proyectos de carácter social, y a la falta de perspectiva que hay en buscar proyectos prácticos.

Analizando carreras de la Facultad de Ingeniería y Arquitectura, la carrera de Ingeniería en Mecatrónica arrojó resultados que en su mayoría son negativos con relación al tema abordado. El $67 \%$ de los entrevistados mencionó que no hay relación entre la carrera que estudian y los proyectos de vinculación que han realizado. El resto afirmó que si existe dicha relación entre la carrera y los proyectos de vinculación realizados.

Los estudiantes de la carrera de Ingeniería en Telecomunicaciones mostraron una opinión dividida en 3 respuestas: NO, SI, Muy pocos. En donde cada una de estas respuestas fue mencionada por un $33 \%$ de los entrevistados. En este sentido podemos constatar que cerca del $66 \%$ de los estudiantes respondieron de forma negativa respecto al tema. Las causas pueden deberse a la dificultad de encontrar organizaciones con problemas vinculadas a esta área del conocimiento, por lo que los proyectos se enfocan más en ámbitos de aportaciones caritativas.

Los comentarios obtenidos de estudiantes de las demás carreras de la Facultad fueron positivos. No obstante, la carrera de Ingeniería en Energía fue una de las pocas en las que el total de los entrevistados mencionó que no hay ninguna relación entre los proyectos que han realizados y su ámbito de estudio. Esto lo atribuyen a la reciente apertura de esta carrera en la universidad.

\subsection{Formatos}

El proceso de vinculación utiliza dos tipos de formatos para recabar información. El primero se titula "Evaluación de calidad del producto entregado en el marco de una asignatura". En éste aparecen tablas con calificación del 1 al 4, en dónde el 1 representa "No satisfactorio", el número 2 representa "Poco satisfactorio", el número 3 significa "Satisfactorio" y el número 4, "Muy satisfactorio".

La información obtenida por medio de este formato permite al Departamento de Vinculación ver el grado de satisfacción de la empresa u organización ante el servicio o 
producto proporcionado por los alumnos de UNITEC. En él se puede analizar el compromiso del docente en el proyecto realizado, así como también el compromiso de los estudiantes y la calidad global de producto presentado. Esta información ayuda al departamento a realizar mejoras para futuros proyectos de vinculación.

El segundo formato titulado "Proyecto de vinculación en el marco de una asignatura", va dirigido al maestro. En él se indica la información general del proyecto realizado, el nombre del proyecto o producto entregado, nombre de la organización beneficiada, nombre de la carrera, asignatura, nombre del docente encargado, y periodo en el que se llevó a cabo el proyecto.

También en él se indica el número de personas beneficiadas por el proyecto y se describe los principales beneficios o aportaciones hechas a las organizaciones por parte de los alumnos involucrados. El docente debe colocar una puntuación con base a 100\%, para determinar el porcentaje del proyecto realizado, y se enlistan los nombres, números de cuenta y cantidad de horas trabajadas de los estudiantes involucrados.

\subsubsection{Limitantes encontradas en los formatos de vinculación.}

Actualmente existen muchas limitantes en cuanto a los formatos utilizados, para lograr una comprensión mucho más precisa del desempeño en la ejecución de los proyectos. Se puede clasificar estas limitantes en externas e internas, las primeras tienen que ver con el uso que se le da al formato, y la segunda, orientada a la estructura y aspectos que se analiza con el mismo.

El primer formato posee limitantes externas las cuales se resumen básicamente en la utilización que se le da a este formato, el cual se supone va enfocada a analizar la calidad los proyectos que se realizan. Lamentablemente, según la información recopilada de las entrevistas a expertos se puede constatar que su análisis es casi nulo. Muchos de los docentes ni si quiera les piden a las contrapartes que llenen el formato, y los que si son aplicados simplemente terminan siendo archivadas, sin un posterior análisis.

En cuanto a las limitantes internas, se puede señalar la falta de una casilla en la cual se situé el nombre del proyecto realizado, lo que ocasiona dificultades para el entendimiento del tipo de proyecto que se llevó a cabo, además de dificultar una posterior clasificación. Otra de las limitantes encontradas es la falta de casilla en donde se ubique el nombre del docente que dirigió el proyecto, a pesar de que este tipo de información ya la posee el departamento de vinculación, sería de ayuda el poder incluirlo en la evaluación para una visión más efectiva y amplia de la persona que estuvo a cargo del proyecto. La falta de un espacio final para sugerencias podría ser otras de las limitantes del formato, en donde la contraparte podría poner sugerencias o comentarios a nivel general en cuanto al proyecto.

Según el análisis realizado de las evaluaciones, se dio a conocer que algunos de los apartados en la sección de aspectos a evaluar son elementos que no siempre tienen relación con los proyectos que se realizan. Por lo tanto, muchas de las respuestas suelen ser "NO 
APLICA" o en algunos casos, las personas dejan en blanco los aspectos a evaluar, por considerar que el aspecto incluido no tiene relación con el proyecto que se realizó.

También se propone agregar más aspectos evaluativos que permitan medir no solo un tipo de proyecto de vinculación, si no, todos los tipos que existen. De esa forma se contará con un formato capaz de evaluar todos los tipos de proyectos que se desarrollan, en cuanto a la satisfacción de la contraparte y calidad de estos. Los aspectos propuestos son los siguientes:

- Compromiso de los estudiantes en el proyecto.

- Presentación del informe final del proyecto.

- Se cumplió con los objetivos del proyecto.

- Aplicabilidad de las soluciones recomendadas del problema abordado.

- Participación y compromiso del docente asignado.

- Calidad global del producto.

A diferencia del primero, el segundo formato se utiliza con más regularidad, pues con él se lleva un registro de los alumnos que participaron en el desarrollo del proyecto. El formato también tiene el objetivo de evaluar el nivel de calidad del proyecto ejecutado desde el punto de vista del docente, y le permite asignar las horas correspondientes a cada estudiante, basándose en el nivel de compromiso y participación que haya observado.

En este caso la principal mejora va orientada a la eliminación de la parte del enlistado de los alumnos participantes. Se considera más adecuado establecer de forma obligatoria la utilización de Excel como medio para procesar dicha información. Este formato se debe orientar a cuestiones relacionadas con el resultado final de proyecto entregado, la calidad del proyecto realizado, así como también conocer información específica relacionada con el proyecto que se realizó y la contraparte involucrada.

\subsection{Calidad de los proyectos de vinculación}

La calidad es un tema de suma importancia para cualquier empresa que presta un servicio o producto, representa la ventaja competitiva más importante de toda empresa, ya que en ella se refleja si los clientes o en este caso beneficiarios, están percibiendo el servicio o producto, como uno de calidad, es decir, si estos cumplen con las expectativas de los clientes.

Para el análisis de la calidad de los proyectos de vinculación, se estudiaron 100 evaluaciones de diferentes organizaciones y tipos de proyectos durante el periodo 2013 al 2017, siendo estudiadas 20 encuestas por cada año. El estudio de las evaluaciones permitió conocer el nivel de satisfacción de las contrapartes en cuantos a los proyectos que han realizado en conjunto con UNITEC. Cabe recalcar la falta de importancia que se le da a estas evaluaciones, muchas de ellas se encuentran incompletas o blanco, con solo el nombre de la organización y el representante de la misma. 
Como se puede apreciar en la siguiente gráfica (Figura 6), un total de 56 evaluaciones de 100 , poseen una calificación perfecta de "Muy Satisfactoria" en todos los apartados evaluados.

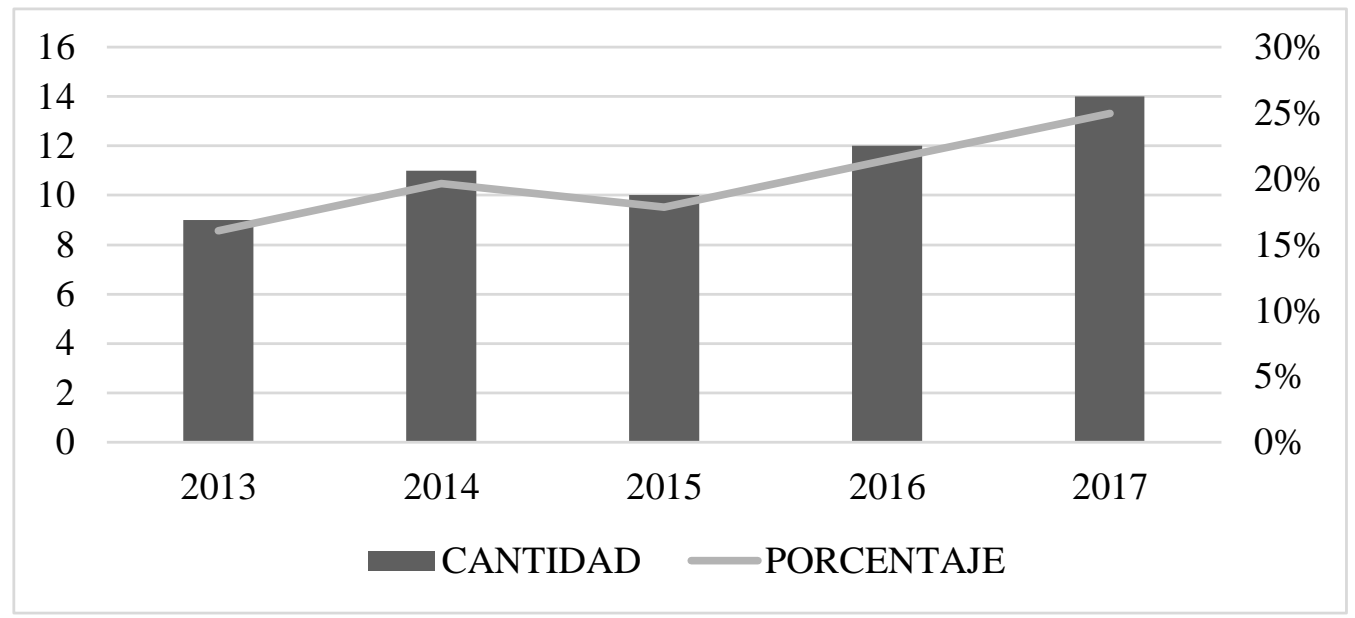

Figura 6. Encuestas con calificación de "Muy Satisfactoria” por año

Fuente: Elaboración Propia

La misma gráfica también muestra la cantidad de evaluación por año con dicha calificación, permitiendo así ver cómo estas han ido aumentando con los años. Se puede apreciar que desde el año 2013 al 2017 las evaluaciones positivas han ido en aumento, con una ligera baja en el año 2015.

Si tomamos en cuenta el total de encuestas con calificación de "Satisfactoria" por año, obtenemos un total de 69 de 100 encuestas con esta calificación, en donde se puede apreciar una baja en el año 2015, pero que continúa de forma ascendente durante los años siguientes.

Por lo que podemos decir que, de las 100 evaluaciones analizadas, 69 tienen una calificación de "Satisfactoria" y "Muy Satisfactoria" y solo 31 evaluaciones obtuvieron calificaciones "Poco satisfactoria" o "No Satisfactoria" en ciertos aspectos.

Las evaluaciones se componen de 5 aspectos principales a evaluar en los proyectos desarrollados. El análisis se realizó por aspecto, para poder entender el desempeño para cada uno de los mismos, y ver la frecuencia de las respuestas.

El primer aspecto a evaluar es el "compromiso de los participantes en el proyecto", en donde el resultado fue el siguiente:

- $86 \%$ "muy satisfecho" por el compromiso que los estudiantes mostraron.

- $12 \%$ dijo estar "satisfecho".

- $1 \%$ mencionó "no estar satisfecho" con el compromiso de los estudiantes.

- $1 \%$ mencionó el aspecto como "no aplicable" en el proyecto desarrollado. 
Entre las causas de la no satisfacción, la contraparte comentó la ambigüedad de las sugerencias que los estudiantes propusieron, la falta de información y su compromiso en la búsqueda de esta.

El siguiente aspecto, "presentación del informe final del proyecto realizado", dio los siguientes resultados:

- $74 \%$ de las contrapartes mencionaron estar muy satisfechas con la presentación del informe final.

- $15 \%$ mencionaron que el aspecto "no aplica" en los proyectos que han realizado.

- $9 \%$ de las contrapartes dio una calificación de "satisfactorio".

- $2 \%$ de las contrapartes mencionaron estar "poco satisfechos" con la presentación del informe final.

Entre las causas de la "no satisfacción", una de las contrapartes mencionó que nunca le presentaron el informe final del proyecto realizado, y que solamente les fueron enviados los resultados de este.

El siguiente aspecto es el de "Aplicabilidad de las soluciones recomendadas del problema abordado":

- 79 por ciento de las contrapartes mencionó sentirse "muy satisfecha" en este aspecto.

- $10 \%$ mencionó que el aspecto "no aplicaba" en el proyecto realizado.

- $6 \%$ corresponde a una calificación "satisfactoria" del aspecto.

- 5\% mencionó estar "poco satisfecho" con el nivel de aplicabilidad de los proyectos.

En términos generales, este fue el aspecto que más poseía calificaciones de "poco satisfactorio". Entre las causas del descontento de algunas de las contrapartes, se mencionó que muchos de los resultados necesitan reformulación, y que los datos no iban de acuerdo al enfoque principal del proyecto.

Algunas de las propuestas para mejorar en este apartado podrían ser el aumentar el nivel de participación y compromiso de los docentes en los proyectos de vinculación, y aumentar el tiempo de desarrollo del proyecto para que los estudiantes puedan absorber todos los conocimientos que necesitan para realizarlo.

El cuarto aspecto a evaluar es la "Calidad global del producto", en donde el $80 \%$ de los encuestados mencionó estar satisfecho por la calidad que presentaron los proyectos de vinculación, un 10\% mencionó estar satisfecho, un $8 \%$ mencionó la no aplicabilidad del apartado en los proyectos que han dirigido y finalmente un $2 \%$ mencionó estar poco satisfecho con la calidad global de los proyectos de vinculación. La causa de la poca satisfacción en este apartado se resume, de forma general, en los problemas surgidos al inicio, desarrollo y finalización del proceso, los cuales han sido detallados en los apartados anteriores. 
Como último apartado en analizar esta la participación y compromiso de la docencia en los proyectos asignados, en donde no se señaló nada negativo o insatisfactorio desde el punto de vista de las contrapartes.

\subsubsection{Análisis de las entrevistas a expertos.}

Como se mencionó anteriormente, en el apartado de calidad también se realizaron entrevistas telefónicas a las contrapartes para poder obtener información más precisa acerca de la calidad de los proyectos y las experiencias de las contrapartes en el desarrollo de los mismos en conjunto con UNITEC.

A cada entrevistado se le preguntó qué calificación le daría al servicio de vinculación de UNITEC, en una calificación del 1 al 5, en donde de forma ascendente, el numero 1 simboliza "Muy insatisfecho" y el 5 simboliza "Muy satisfecho", el 90\% de los entrevistados calificó el servicio con un 5, mientras que el 10\% restante lo calificó con un 4.5 , 4, etc. Obteniendo un promedio de 4.5 de 5 . Con el objetivo de poder indagar acerca de las causas de esta baja calificación, se les preguntó a los entrevistados, qué aspectos o elementos negativos propiciaron la calificación dada.

Una de las entrevistas fue realizada al representante de "CASM", una ONG que contribuye activamente en el fortalecimiento de los procesos de democratización y de desarrollo local y regional de Honduras, encargada básicamente, de identificar problemas o necesidades latentes en la sociedad y buscar algún tipo de solución vinculando o buscando organizaciones que la apoyen en esto. Él mencionó un proyecto realizado en la empresa Vivero "El esfuerzo". El proyecto fue desarrollado por alumnos de la carrera de Diseño Gráfico, y consistía básicamente en la elaboración de la parte gráfica de la compañía, la elaboración de logos, gráficos, imágenes, entre otros elementos. Entre los problemas encontrados, la contraparte mencionó que les hicieron observaciones a los resultados finales proporcionados por los estudiantes, pero estas correcciones nunca fueron implementadas o entregadas, por lo que la entrega del proyecto quedo incompleta.

El representante de "CASM" no fue el único que mencionó este problema, Melvin Díaz, representante de Fundación Children International, también comentó acerca de este problema. En este sentido ambos entrevistados mencionaron el tiempo de los trimestres como una limitante para la realización correcta de estos proyectos, puesto que existen ocasiones en las que los estudiantes no tienen el tiempo suficiente para recopilar toda la información necesaria para el desarrollo de los mismos, incidiendo en la baja calidad de los mismos, y en la aparición de problemas o fallas que no siempre se logran corregir.

El representante de "CASM" también mencionó el compromiso de los docentes en los proyectos de vinculación, como otro apartado a mejorar, debido a que un docente mucho más presente y en constante supervisión de los trabajos realizados, debería lograr incidir en el aumento de la calidad de los mismo, puesto que cualquier problema que va surgiendo, va siendo analizado y corregido en el momento, por una persona que se supone tiene mucha más experiencia en el campo. 
Como recomendaciones finales los entrevistados proponen el establecimiento de forma definitiva de los proyectos en las clases seleccionadas de la carrera, es decir, que cada clase tenga ya establecido el proyecto que se realizará. También se propone la subdivisión de los proyectos en etapas, de tal manera que los proyectos reciban continuidad y no terminen abandonados.

Otra de las entidades entrevistadas, fue la Municipalidad de San Pedro Zacapa, Santa Bárbara, la cual desarrolló, con la ayuda de UNITEC, proyectos relacionados con la programación del sistema de agua potable de la comunidad, la creación de una planta purificadora de agua, diseño de planos, entre otros proyectos. Los mismos fueron elaborados con la ayuda de los estudiantes de las carreras de Ingeniería Civil y Arquitectura.

El entrevistado mencionó que todos los proyectos han sido llevados a cabo con éxito, pero indicó que, en una ocasión, durante la entrega final del proyecto, faltó entregar un documento específico que aludía al cálculo de materiales del proyecto, el entrevistado mencionó que el documento era requerido por la municipalidad, pero nunca fue entregado por UNITEC.

En este sentido el entrevistado propone mejoras que apunten a la facilitación de estos documentos, además del establecimiento desde un inicio, de los entregables del proyecto. Entre otros aportes mencionó el de promover el compromiso de los estudiantes en los proyectos realizados, así como también la amplificación del abanico de servicio de vinculación.

Los demás entrevistados mencionaron que los proyectos son de muy alta calidad. Hasta ahora todos los proyectos que se han realizado han cumplido con los objetivos a cabalidad, a excepción de un entrevistado que expresó que, al principio, los objetivos del proyecto no se estaban cumpliendo, pero que, al continuar trabajando con UNITEC, más adelante, se pudieron desarrollar de mejor forma.

En cuestión de la aplicabilidad de los proyectos, los entrevistados mencionaron que la mayoría de éstos se están utilizando en la actualidad. Por ejemplo, el Teléfono de la Esperanza integró un sistema de perfiles de puestos de trabajo realizado por UNITEC, y la Municipalidad de San Pedro Zacapa ha utilizado los diseños, planos e implementado estudio acuíferos desarrollados por los estudiantes de la universidad.

\section{Conclusiones}

Se indagó acerca de las limitantes del proceso de vinculación universitaria en la parte interna que abarca los procesos administrativos, desarrollo del proyecto y actividades posteriores a la finalización del mismo. Entre las observaciones más importantes está la falta de una etapa de análisis para las evaluaciones de calidad que se realizan a las 
contrapartes, también se descubrió diferentes etapas como la de inclusión en el sílabo, análisis de los proyectos, entre otras; como etapas con bajo compromiso y enfoque; y que inciden en el incorrecto funcionamiento del sistema. También se realizaron entrevistas a los estudiantes de la universidad en donde se dio a conocer que, durante el desarrollo de estos proyectos, el tiempo, el transporte y la inseguridad son factores que más afectan el sistema.

Se analizó el nivel de calidad de los proyectos de vinculación, el cual produjo un resultado satisfactorio, cerca del $80 \%$ de las evaluaciones analizadas consideraron de forma global el servicio como excelente, además de eso, según los representantes de las contrapartes entrevistadas, el promedio de satisfacción del servicio es de 4.5/5. Muchas de las contrapartes mencionaron que la calidad es excepcional, pero que aspectos como la aplicabilidad de los proyectos afectan la calidad del mismo, entre las propuestas de mejora en este apartado, mencionaron el tiempo como la causa de la baja aplicabilidad de los proyectos, ya que los estudiantes empiezan a desarrollar los proyectos en la segunda o tercera semana de clases, por lo que no llegan a recopilar la información necesaria para un correcto desarrollo y aplicabilidad del mismo. Otro de los factores que se descubrió afectan la calidad del producto, es en la presentación final del proyecto en donde los entrevistados mencionaron que siempre que existe la oportunidad, ellos buscan realizar correcciones a los proyectos, con el fin de ayudar en el aprendizaje de los estudiantes, así como también en el resultado final del proyecto, ellos destacaron que no siempre se corrigen las observaciones realizadas, debido a factores de tiempo, por lo que el proyecto queda incompleto, lo que afecta la calidad global del mismo.

Con base en el análisis de los resultados, se concluye que la Universidad Tecnológica Centroamericana (UNITEC), es una institución que se encuentra siempre a la vanguardia buscando destacar en todo momento y se ubica muy por encima de la mayoría de las universidades de la región en cuanto a vinculación universitaria, existen muy pocos aspectos como la estructura, enfoque, formatos de postulación de proyectos, alcance en las cuales universidades como la UNAH y la UPNFM resultan ventajosa, lo que podría deberse a la antigüedad y apoyo por parte del Gobierno, en términos de universidades privadas, UNITEC se encuentra en primer puesto.

\section{Bibliografía}

Alcantara, G. A. (2007). Misión social y modelos de extensión . Revista Iberoamericana de Educación, 3.

ANSI. (2016). Normas American National Standars Institute (ANSI). Washington.

Baez, L. (1992). Planeación y extensión universitaria. Revista de la Educación Superior, 81.

Bernheim, C. T. (1998). La reforma universitaria de Córdoba. Educación Superior y Sociedad Vol 9, 103-127. 
Bernheim, C. T. (2003). LA UNIVERSIDAD ANTE LOS RETOS DEL SIGLO XXI. Yucatán: Ediciones de la Universidad Autónoma de Yucatán.

Bernheim, C. T. (2003). LA UNIVERSIDAD LATINOAMERICANA ANTE LOS RETOS DEL SIGLO XXI. México, DF.

Buchbinder, P. (2006). La Universidad: breve introducción a su evolución histórica. Universidad Nacional del Litora.

Chang, R. Y., \& Niedzwiecki, M. E. (1999). Las herramientas para la mejora continua de la calidad Volumen 2. Buenos Aires: Ediciones Granica.

Consejo de educación superior. (29 de 09 de 2017). portalunico. Obtenido de http://portalunico.iaip.gob.hn/Archivos/UPNFM/Regulaciones(normativa)/Leyes/2015/L ey\%20de\%20Educacion\%20Superior.pdf

D'Andrea, R. E., Zubiría, A. Sastre Vásquez, P. (2014). RESEÑA HISTÓRICA DE LA EXTENSIÓN UNIVERSITARIA. Buenos Aires: Jornada de la Extensión del Mercosur.

Dirección de Educación Superior. (29 de 09 de 2017). UNAH. Obtenido de file://CC:/Users/JISAAC/Downloads/NORMAS-ACADÉMICAS-DE-LA-EDUCACIÓNSUPERIOR.pdf

García Garrido, J. (1999). La universidad del siglo XXI. Lección inagural del curso 1999- 2000.

García Matías, F., Morales Soto, M., \& Castellanos Suarez, J. A. (2009). EXTENSIÓN Y VINCULACIÓN UNIVERSIDAD-SOCIEDAD, CONCEPTOS INACABADOS. Investigación Agropecuaria, 99.

González, G. R., \& González, M. (2006). Extensión universitaria: una aproximación conceptual desde la perspectiva cubana. Revista Cubana de Educación Superior, 69-76.

La Gaceta. (1999). Periódico oficial de la República de Honduras. Tegucigalpa, Honduras.

Menéndez, G. (2014). DESARROLLO Y CONCEPTUALIZACIÓN DE LA EXTENSIÓN UNIVERSITARIA. Santa Fe.

Miranda Gonzáles, F. J., Chamorro Mera, A., \& Rubio Lacoba, S. (2007). INTRODUCCIÓN A LA GESTIÓN DE LA CALIDAD. Madrid: Delta Publicaciones.

Murillo, O. D. (2017). El movimiento estudiantil de Cordoba y su influencia en Honduras. Historia de la educación Latinoamericana, 37-52.

Nacional, C. (02 de 10 de 2017). FAOLEX. Obtenido de http://extwprlegs1.fao.org/docs/pdf/hon94981anx.pdf

Ortiz-Riaga, M. C., \& Morales-Rubiano, M. E. (2011). La extensión universitaria en América Latina: concepciones y tendencias. Educación y Educadores, 349-366.

Rivero, A., Luis, J., M, C., \& R, J. (2003). La Extensión Universitaria y su relación con la Educación Comunitaria. Revista de educación superior.

Salgado, R. (2000). La educación superior en Honduras. Tegucigalpa. 
UNAH. (2 de 10 de 2017). DIRECCIÓN DE VINCULACIÓN UNIVERSIDAD- SOCIEDAD. Obtenido de https://vinculacion.unah.edu.hn/sobre-nosotros/funciones-de-los-comites-devinculacion/

UNAH. (2017). DISPOSIONES COMUNES DE LEYES Y REGLAMENTOS DE LA UNAH SOBRE EL QUEHACER ACADÉMICO DE VINCULACIÓN CON LA SOCIEDAD. TEGUCIGALPA.

UNESCO. (1998). Conferencia Mundial sobre la Educación Superior., (pág. 89). DF.

UNICAH. (05 de 10 de 2017). UNICAH. Obtenido de http://blogs.unicah.edu/spsp/?page_id=395

UNITEC. (11 de 10 de 2017). Reglamento Académico de UNITEC. Obtenido de http://matricula.unitec.edu/ReglamentoAcademico.pdf

UNITEC. (11 de 10 de 2017). Reglamento de Vinculación y Servicio Social. Obtenido de http://www.unitec.edu/assets/pdf/reglamentos-vida-estudiantil//reglamento-devinculacion-y-servicio-social.pdf 